\title{
Computerized patient reported symptom assessment in radiotherapy: a pilot randomized, controlled trial
}

\author{
Erik K. Fromme, MD, MCR, FAAHPM, \\ Mail Code: L586, Division of Hematology \& Medical Oncology, OHSU Knight Cancer Institute, \\ Oregon Health \& Science University, Portland, OR 97239, Phone: 503-494-3152, Fax: \\ 503-494-3257, frommee@ohsu.edu
}

\author{
Emma B. Holliday, MD, \\ Division of Radiation Oncology, The University of Texas MD Anderson Cancer Center, 1515 \\ Holcombe Blvd Unit 97, Houston, Texas 78240, Phone: 210-854-5399, \\ emmaholliday@gmail.com
}

Lillian M. Nail, PhD, RN,

School of Nursing, Oregon Health \& Science University

Karen S. Lyons, PhD,

School of Nursing, Oregon Health \& Science University

Michelle R. Hribar, PhD, and

Department of Medical Informatics and Clinical Epidemiology, Oregon Health \& Science University

\section{Charles R. Thomas Jr, MD}

Department of Radiation Medicine, OHSU Knight Cancer Institute, Oregon Health \& Science University

\begin{abstract}
Purpose-Computer-based, patient-reported symptom survey tools have been described for patients undergoing chemotherapy. We hypothesized that patients undergoing radiotherapy might also benefit, so we developed a computer application to acquire symptom ratings from patients and generate summaries for use at point of care office visits and conducted a randomizedcontrolled pilot trial to test its feasibility.

Methods-Subjects were randomized prior to beginning radiotherapy. Both control and intervention group subjects completed the computerized symptom assessment, but only for the intervention group were printed symptom summaries made available before each weekly office visit. Metrics compared included the Global Distress Index (GDI), concordance of patient-reported symptoms and symptoms discussed by the physician and numbers of new and/or adjusted symptom management medications prescribed.
\end{abstract}

Correspondence to: Erik K. Fromme.

Presented in part at: 2010 International Society for Quality of Life Research Annual Meeting in London, England, and the 2011 American Society for Therapeutic Radiation Oncology Annual Meeting in Miami, Florida.

Conflict of Interest: The authors declare that they have no conflict of interest. 
Results-112 patients completed the study: 54 in the control and 58 in the intervention arms. There were no differences in GDI over time between the control and intervention groups. In the intervention group, more patient-reported symptoms were actually discussed in radiotherapy office visits: $46 / 202$ vs. $19 / 230$. A sensitivity analysis to account for within-subjects correlation yielded $23.2 \%$ vs. $10.3 \%$ ( $\mathrm{p}=.03$ ). Medications were started or adjusted at $15.4 \%$ (43/280) of control visits compared to $20.4 \%(65 / 319)$ of intervention visits $(\mathrm{p}=.07)$.

Conclusions-This computer application is easy to use and makes extensive patient-reported outcome data available at the point of care. Although no differences were seen in symptom trajectory, patients who had printed symptom summaries had improved communication during office visits and a trend towards more active symptom management during radiotherapy.

\section{Keywords}

patient-reported outcomes; supportive care; radiation therapy; communication

\section{Introduction}

A myriad of symptoms may be associated with cancer and compound the psychological and emotional toll a cancer diagnosis exerts on a patient and their loved ones. Approximately half of all cancer patients report pain, of whom approximately one third rate their pain as moderate or severe [1]. Non-pain related symptoms including fatigue, mucositis and nausea/ vomiting likewise afflict many cancer patients and require active management in order to prevent a significant decline in quality of life [2]. Cancer treatment can often exacerbate existing symptoms or cause toxicities resulting in new or worsening symptoms [3]. With the increasing frequency of multi-modality regimens, optimizing patient-provider communication is a challenge to effectively recognizing and managing symptoms.

Previous research has shown that, although clinicians believe symptom management is important, they are often unaware of patients' symptoms. When patient-reported symptoms using the Quality of Life (QOL) Questionnaire QLQ-C30 were compared with concurrent physician-reported symptoms in the Adverse Event logs of a clinical trial, physicians were unaware of half the symptoms patients reported experiencing. Even more surprising was that half of the symptoms physicians were documenting were not reported by patients [4]. Inadequate management can result when physicians fail to recognize patient symptoms [5], so measures taken to increase mutual understanding between physician and patient has the potential to greatly improve patient QOL during treatment.

Symptom and QOL assessment research has yielded valid and reliable measures of patient experience[6][7][8], as well as strategies to best acquire and utilize this information in a clinical encounter [9]. Early work in this area has focused almost exclusively on oncology patients receiving chemotherapy [10][11][12]. Computer applications focused on chemotherapy patients' symptoms and quality of life have been shown to improve communication, mental health outcomes, and QOL [13][14][15][16]. Such applications have not yet been validated for patients undergoing radiotherapy, and herein lies a gap in clinical care that merits attention. Up to two-thirds of all cancer patients will receive some form of radiotherapy. Additionally, radiotherapy-related symptoms can be affected by the anatomic 
site, target volume, technique, dose, fractionation and duration of radiotherapy course, as well as the receipt of concurrent or neoadjuvant systemic therapies [17][18][19][20]. Severe toxicity during radiotherapy can lead to the need for unplanned treatment breaks or premature cessation of therapy. This can have adverse radiobiologic consequences such as accelerated tumor cell repopulation [21] and adverse clinical consequences such as an estimated $1 \%$ decrease in tumor control with every day radiation is delayed [22].

To investigate the utility of computer-based patient-reported symptom surveys and summaries in the clinical setting, we developed a computer application that allows patients to electronically self-report their current symptoms and generates a printed report summarizing these data that the physician and patient can use in their weekly on-treatment visit (OTV). We hypothesized that providing these reports could improve symptom management outcomes for radiotherapy patients as measured by increased concordance in physician-patient communication, increased attempts by physicians to manage symptoms, or lower total symptom burden. To test this, we designed a pilot single-institution randomized, controlled trial.

\section{Methods}

\section{Developing and testing the computer application}

We adapted a graphic user interface, previously developed for elderly patients receiving chemotherapy [23], to run on a Windows-based PC. Question items determined through focus groups and interviews with radiation oncologists included: Karnofsky Performance Status [24], overall QOL, pain intensity in nine anatomic sites, 32 common cancer symptoms using both the Memorial Symptom Assessment Scale (MSAS)-Short Form [6] [25][26] and zero to ten Numeric Rating Scales [27], three skin damage items, the physical function scale from the EORTC QLQ-C30[28], and up to 12 items specific to 13 different cancers. We conducted pilot testing of 12 patients receiving radiotherapy to determine visual preference of graphic display and question architecture. Designed to run as a series of web pages, subjects used a touch screen monitor to input their responses (Figure 2). The application then printed a report summarizing current symptoms and up to 3 previous time points (Figure 3). To measure patient-reported ease of use we employed Davis' validated 8item instrument designed for computerized questionnaires [29].

\section{Testing the computer application in a randomized pilot study}

Approval was obtained from the Institutional Review Board to carry out this pilot study. The aims were to demonstrate feasibility and generate the preliminary data necessary to obtain effect size estimates for the intervention on the polynomial slope coefficients in a randomeffects regression model and subsequently design a fully powered randomized-controlled trial. A sample size of 100 was selected for this pilot study based on i.) feasibility, ii.) the ability to determine the appropriate polynomial model (i.e., linear versus quadratic) and iii.) give stable estimates of the parameter values (i.e. for the random effects regression) with allowances for attrition over time. 
Informed consent was obtained from all individual participants included in the study. The protocol is available in the Supplemental Materials. Subjects were enrolled prior to starting a new radiotherapy regimen for cancer and were randomized at the time of enrollment using blinded envelopes. Only patients with treatment regimens $\geq 4$ weeks were recruited. The CONSORT Diagram is shown in Figure 1. After enrollment but prior to initiation of radiation, patients were asked to fill out a questionnaire inquiring as to their level of education, employment status, whether or not they had ever used a touch-screen computer, how much computer experience they had (self-reported on a 5-point Likert Scale from $1=$ "none at all" to 5 = "very much"), and whether or not they would be willing to complete a survey as part of their usual care. Intervention group subjects completed the computerized symptom assessment and received a printed symptom summary before their weekly OTV with their radiation oncologists, who also received a copy of the summary. Control group subjects completed the same assessment, though printed symptom summaries were not provided to the patients or physicians. Intervention and control group subjects filled out the computerized symptom assessments in designated patient education cubicles just adjacent to the patient waiting area that were outfitted with touchscreen computers. Patients could request assistance reading the assessment verbiage but could not receive further explanation or clarification by research or clinic staff when answering questions.

\section{Symptom Quantification}

The MSAS Global Distress Index (GDI) was used to collect and compare symptom data and is a reliable and valid measure consisting of the mean score of 10 items, including four psychological symptoms (sadness, worry, irritability, and nervousness) and six physical symptoms (lack of appetite, lack of energy, feeling drowsy, constipation, dry mouth, and pain) [26]. Additional outcomes collected and compared included physician-patient concordance in communication about symptoms and the total number of symptom management prescriptions written by the radiation oncologists (a surrogate measure of overall efforts to manage symptoms). Concordance in physician-patient communication for symptoms was assessed by audio-recording the $2^{\text {nd }}$ or $3^{\text {rd }}$ OTV for a subset of patients. Recordings were coded by a blinded observer to identify every mention of a symptom by the physician, patient, or family member during the OTV. These mentioned symptoms were compared to the symptoms reported by the patient. Symptom management prescriptions were tracked in the patient's electronic medical record and included medications in the following classes: analgesics, muscle relaxers, steroids, antacids, anti-emetics, appetite stimulants, laxatives, anti-diarrheals, anti-depressants or anti-psychotics, sedative/hypnotics, skin care or mouth care products, and urinary or erectile dysfunction medications.

\section{Data Analysis}

Pearson's Chi-Squared test was used for between-group comparisons of categorical variables, and Student's t-test was used for between-group comparisons of continuous variables. All $P$ values reported are two-sided. The program Hierarchical Linear Modeling (HLM) 7 was used to examine the first four GDI scores over time. HLM involves data that vary at two levels: within individuals (level one) and between individuals (level two). At level one, each patient's change in distress is represented by a trajectory captured by a set of unique parameters: intercept and slope. At level two, these unique parameters become 
outcome variables to be explained by a set of predictors. The first step was to fit a level one model to capture the average pattern of change over time in the sample and the variance around the average. If significant variation existed, a level two model was examined to compare GDI scores across OTVs between the intervention and control groups, controlling for patient age, gender, and receipt of concurrent chemotherapy.

For the audio-recorded office visit, we restricted our analysis to the 43 symptoms included in the computer application for all subjects. Concordance meant that a symptom selfreported by the patient using the computerized symptom assessment was mentioned or discussed in the office visit. We created Venn Diagrams to illustrate the degree of concordance for patients in the intervention and control groups, and performed sensitivity accounting for within-subjects correlation.

\section{Results}

\section{Patient and treatment characteristics}

We approached 145 patients, $83 \%$ consented, and 77\% participated for a total of 112 patients, including 54 control and 58 intervention subjects. In total, 109 (97.3\%) patients received radiotherapy with curative intent. Table 1 describes the demographics of the control and intervention groups. Although not stratified by these characteristics, there were few differences between the control and intervention groups. However, significantly more patients in the intervention arm received concurrent chemotherapy (25 (43.1\%) vs 13 $(24.5 \%) ; \mathrm{p}=.04)$. Table 2 describes the radiotherapy details for the patients in the control and intervention groups.

\section{Completion of the survey instrument}

Subjects completed the computerized questionnaire in an average of 10.5 minutes (range 424 minutes). Ease-of-use was rated positively with an average score of $+11.9(\mathrm{SD}=4.1)$ on a scale of -16 to +16 , with -16 indicating hardest to use and +16 indicating easiest to use.

\section{Overall symptom burden}

For the entire cohort, pain was reported at $80.3 \%$ of all clinic visits; moderate and severe pain were reported at $34.9 \%$ and $12.1 \%$ of all clinic visits, respectively. Other common symptoms were lack of energy (reported at $60.3 \%$ of visits), dry mouth (35.3\%), difficulty sleeping (34.4\%), lack of appetite (30.6\%), urination problems (28.1\%), nausea $(21.3 \%)$, constipation (20.3\%), shortness of breath (11.8\%), and weight loss (13.1\%). The mean \pm SD GDI scores from the control vs intervention groups for weeks 1, 2, 3 and 4 are presented in Table 3 .

HLM analysis found that a quadratic (non-linear) model fit the GDI data significantly better than a linear model $(\mathrm{p}<.001)$. The average slope was not significantly different from zero, but there was significant variability around the average slope $(\mathrm{p}<.001)$ indicating some patients increased in distress and others decreased over time. This significant variability appears to have led to an average change close to zero, reinforcing the need to use methods such as HLM that examine such variability rather than a traditional average score. 
Comparing GDI as a measure of overall symptom burden between the intervention and control groups, the full level two HLM regression model with control vs. intervention group, age, gender and chemo status included provided a significantly better fit to the data over an unconditional model with no predictors ( $\mathrm{p}<.01)$. The intervention, however, did not produce significant improvement in GDI over time. Receiving chemotherapy was significantly associated with higher distress scores at baseline only $(\mathrm{p}<.001)$. On the other hand, patient age was a significant predictor of change with younger patients experiencing faster increases in distress over time ( $\mathrm{p}<.05)$ (Table 4).

\section{Communication concordance}

49/112 (43.8\%) subjects agreed to have one of their OTVs audio-recorded including 26 in the control group and 23 in the intervention group. Concordance was significantly higher in the intervention group, with more symptoms reported by subjects using the computerized questionnaire being discussed in the subsequent office visit: 46/202 vs. 19/230. Using sensitivity accounting for within subjects correlation yielded $23.2 \%$ vs. $10.3 \%(\mathrm{p}=.03)$. The most common symptoms that were reported by patients on the questionnaire but not subsequently discussed during the OTV were: difficulty sleeping (Control: 12/211 (5.7\%), Intervention: 8/156 (5.1\%), Overall: 20/367 (5.5\%)) and pain in the neck or shoulders (Control 10/211 (4.7\%), Intervention 10/156 (6.4\%), Overall: 20/367 (5.5\%)). Conversely, the most common individual symptoms that were discussed during the OTV but not previously reported on the questionnaire were: lack of energy (Control: 7/92 (7.6\%), Intervention: 7/59 (11.9\%), Overall: 14/151 (9.3\%)) and pinkness of skin (Control: 9/92 (9.8\%), Intervention: 5/59 (8.5\%), Overall: 14/151 (9.3\%)). There were no significant differences in symptoms reported but not discussed or vice versa between the control and intervention groups. The Venn Diagrams in Figure 4 show the relationship between reported and discussed symptoms.

\section{Symptom management}

A new medicine was started or a dose adjusted at only $15.4 \%$ (43/280) of control visits compared to $20.4 \%(65 / 319)$ of intervention visits ( $\mathrm{p}=.07)$. Pain management prescriptions were written or adjusted at $9.5 \%$ of visits overall

\section{Discussion}

This randomized, controlled pilot trial examined the longitudinal impact of providing patient-reported symptom summaries to radiotherapy patients and their clinicians in realtime. The intervention did not result in improved trajectory of patient symptom burden as measured by the GDI. However, for intervention arm patients, there was increased concordance between symptoms reported by the patients on computerized forms and symptoms discussed between the patient and physician at the weekly OTV. Additionally, there was a trend observed towards increased physician symptom management prescribing for patients in the intervention arm.

Patient reported outcomes (PROs) are of increasing interest both in the clinical trial [30] as well as the clinic [31] settings. Computer-based symptom surveys have been described and 
evaluated as a means of utilizing technology to better facilitate gathering PRO data and delivering it to the care team for clinical decision making [13][14][15][16]. In order for a PRO survey to be effective, it must be easy for the patient to complete in a timely manner prior to their clinic visit. Subjects in our trial were able to complete the survey instrument in an average of 10.5 minutes, suggesting it could realistically be completed in the waiting room or exam room prior to the OTV. The majority of patients in both the control and intervention arms stated they would be willing to complete a similar survey in future clinical interactions, indicating barriers to patient "buy-in" to this process would likely be low.

Several lines of evidence suggest that receipt of printed symptom summaries may indeed improve physician practices in the radiation oncology clinic. Providing physicians with paper-based reminders during office visits has been shown to be an effective strategy for improving physician practices over several clinical conditions [32]. By sharing the printed symptom summaries with both the physician and the patient, it becomes a concrete platform for further discussion of symptoms and an aid to shared decision-making, which have also been suggested to positively influence physician practices [33]. It stands to reason that management of symptoms during cancer therapy is best accomplished when the treating physician and patient agree on the presence and severity of a patient's symptoms. Using discussion about patient-reported symptoms as a measure of this concordance, we found a paucity of overlap between the symptoms discussed and reported. The intervention group showed both an increase in symptom discussion concordance and the total number of symptoms discussed, suggesting that the printed summary is a useful tool in furthering this goal. We found that sleep disturbances as well as neck and shoulder pain were reported by patients in the questionnaire but no subsequently discussed with their physicians, while discussion of decreased energy and skin reaction were more likely to be discussed during an OTV even when these symptoms weren't reported by the patient. In a fully-powered trial, these trends could provide valuable insight regarding symptoms experienced by patients and not routinely brought in discussions with their health care providers as well as those that are addressed in the clinical discussion setting but might be missed by existing PRO questionnaires.

Increased discussion of patient-reported symptoms alone is not sufficient to improve symptom control outcomes unless the discussion is translated into action. However, studies have shown physicians who are made aware of patient-reported symptoms are more likely to act [11]. These results are consistent with our findings; intervention group patients received $32 \%$ more symptom management prescriptions than control group patients. Because symptom management prescribing was infrequent, this result did not reach statistical significance ( $\mathrm{p}=0.07$ ) but would likely be significant in a fully powered study. Additionally, although we collected data regarding new and adjusted symptom management prescription medication, we did not capture data regarding referrals to other physicians or providers for symptom management including social work, nutrition, physical or occupational therapy, lymphedema therapy, speech and swallow therapy, nor did we capture discussion or recommendation of non-prescription medications for symptom control including. Therefore, we potentially underestimated the discussion of efforts taken to improve patient symptoms and improve QOL. Ultimately, we did not show a measurable improvement in patient symptoms as a result of our intervention. These results largely fall in line with other 
published results as well as a meta-analysis of electronic symptom reporting studies [34]. Although improved patient-physician communication is a noble goal, in planning future studies, emphasis should be placed on identifying interventions that lead to a measurable and meaningful improvement in patient symptoms.

This study provides a novel and valuable analysis on the effects of implementing a system of administering a computer-based patient-reported symptom survey and providing the patient and radiation oncologist with the resulting real-time feedback. However, it is not without limitations. This study involved a single institution and may not accurately reflect the practice patterns of physicians at other institutions. Second, this was a pilot study and thus not intentionally powered to produce significant results because effect sizes were not yet known. Although the control and intervention groups were relatively well-balanced, the groups were not stratified with regard to patient and treatment characteristics. More patients in the intervention group had head and neck or colorectal tumors, and more patients in the intervention group received concurrent chemotherapy. Both tumor location and concurrent therapy can affect the side effects of radiotherapy, and may have also affected symptom discussion during OTVs as well as symptom management medication prescribing. Finally, we did not collect information regarding any differences in length of OTVs between the control and intervention groups. This would be an important metric to include in future studies as it would provide information as to whether computer-based symptom surveys and real-time feedback make clinic visits more efficient. Additionally, such data would conversely identify whether the implementation of this instrument requires significantly more provider time for each visit. Study strengths include the randomized controlled design, rigorous symptom measurement and multilevel modeling analysis, and innovative approach to assessing communication and symptom management practices.

In conclusion, this pilot study demonstrates that a computer-based patient-reported symptom survey can be feasibly administered to cancer patients receiving radiotherapy prior to their weekly OTV with their treating physician. Additionally, our results suggest that printed summaries of patient-reported symptoms improves concordance between symptoms experienced by the patient and issues discussed during the office visit, and this improved communication may lead to more active symptom management. Although the printed symptom summary did not result in improved trajectory of patient symptom burden, taken comprehensively, these findings suggest that this technology has real potential to improve patient outcomes if incorporated as part of a multi-modal intervention. Future efforts will include implementation of a multi-center trial powered to answer the questions of whether or not patient symptom burden can be improved by utilization of this patient-reported symptom survey and providing real-time feedback to both patients and physicians.

\section{Supplementary Material}

Refer to Web version on PubMed Central for supplementary material.

\section{Acknowledgments}

The authors would like to thank Tawni Kenworthy Heinige and Brie Noble for assistance preparing this manuscript.

Support Care Cancer. Author manuscript; available in PMC 2017 April 01. 
Funding: This project was supported by R21CA141172 from the National Cancer Institute.

\section{References}

1. Van den Beuken-van Everdingen MHJ, de Rijke JM, Kessels AG, et al. Prevalence of pain in patients with cancer: a systematic review of the past 40 years. Ann Oncol Off J Eur Soc Med Oncol ESMO. 2007; 18:1437-1449.

2. Von Gunten CF, Gafford E. Treatment of non-pain-related symptoms. Cancer J Sudbury Mass. 2013; 19:397-404.

3. Reilly CM, Bruner DW, Mitchell SA, et al. A literature synthesis of symptom prevalence and severity in persons receiving active cancer treatment. Support Care Cancer Off J Multinatl Assoc Support Care Cancer. 2013; 21:1525-1550.

4. Fromme EK, Eilers KM, Mori M, et al. How accurate is clinician reporting of chemotherapy adverse effects? A comparison with patient-reported symptoms from the Quality-of-Life Questionnaire C30. J Clin Oncol Off J Am Soc Clin Oncol. 2004; 22:3485-3490.

5. Cleeland CS, Gonin R, Hatfield AK, et al. Pain and its treatment in outpatients with metastatic cancer. N Engl J Med. 1994; 330:592-596. [PubMed: 7508092]

6. Chang VT, Hwang SS, Feuerman M, et al. The memorial symptom assessment scale short form (MSAS-SF). Cancer. 2000; 89:1162-1171. [PubMed: 10964347]

7. Cleeland CS, Mendoza TR, Wang XS, et al. Assessing symptom distress in cancer patients: the M.D. Anderson Symptom Inventory. Cancer. 2000; 89:1634-1646. [PubMed: 11013380]

8. Groenvold M, Klee MC, Sprangers MA, Aaronson NK. Validation of the EORTC QLQ-C30 quality of life questionnaire through combined qualitative and quantitative assessment of patient-observer agreement. J Clin Epidemiol. 1997; 50:441-450. [PubMed: 9179103]

9. Frost MH, Bonomi AE, Cappelleri JC, et al. Applying quality-of-life data formally and systematically into clinical practice. Mayo Clin Proc. 2007; 82:1214-1228. [PubMed: 17908528]

10. Detmar SB, Muller MJ, Schornagel JH, et al. Health-related quality-of-life assessments and patient-physician communication: a randomized controlled trial. JAMA. 2002; 288:3027-3034. [PubMed: 12479768]

11. Ruland CM, White T, Stevens M, et al. Effects of a computerized system to support shared decision making in symptom management of cancer patients: preliminary results. J Am Med Inform Assoc JAMIA. 2003; 10:573-579. [PubMed: 12925545]

12. Velikova G, Booth L, Smith AB, et al. Measuring quality of life in routine oncology practice improves communication and patient well-being: a randomized controlled trial. J Clin Oncol Off J Am Soc Clin Oncol. 2004; 22:714-724.

13. Basch E, Artz D, Dulko D, et al. Patient online self-reporting of toxicity symptoms during chemotherapy. J Clin Oncol Off J Am Soc Clin Oncol. 2005; 23:3552-3561.

14. Basch E, Artz D, Iasonos A, et al. Evaluation of an online platform for cancer patient self-reporting of chemotherapy toxicities. J Am Med Inform Assoc JAMIA. 2007; 14:264-268. [PubMed: 17329732]

15. Basch EM, Reeve BB, Mitchell SA, et al. Electronic toxicity monitoring and patient-reported outcomes. Cancer J Sudbury Mass. 2011; 17:231-234.

16. Aktas A, Hullihen B, Shrotriya S, et al. Connected Health: Cancer Symptom and Quality-of-Life Assessment Using a Tablet Computer: A Pilot Study. Am J Hosp Palliat Care. 2013

17. Bradley N, Davis L, Chow E. Symptom distress in patients attending an outpatient palliative radiotherapy clinic. J Pain Symptom Manage. 2005; 30:123-131. [PubMed: 16125027]

18. Sheu T, Fuller CD, Mendoza TR, et al. Nomogram for predicting symptom severity during radiation therapy for head and neck cancer. Otolaryngol--Head Neck Surg Off J Am Acad Otolaryngol-Head Neck Surg. 2014; 151:619-626.

19. Dehing-Oberije C, De Ruysscher D, Petit S, et al. Development, external validation and clinical usefulness of a practical prediction model for radiation-induced dysphagia in lung cancer patients. Radiother Oncol J Eur Soc Ther Radiol Oncol. 2010; 97:455-461.

20. Valdagni R, Rancati T, Fiorino C. Predictive models of toxicity with external radiotherapy for prostate cancer: clinical issues. Cancer. 2009; 115:3141-3149. [PubMed: 19544543] 
21. Tarnawski R, Fowler J, Skladowski K, et al. How fast is repopulation of tumor cells during the treatment gap? Int J Radiat Oncol Biol Phys. 2002; 54:229-236. [PubMed: 12182996]

22. Bese NS, Hendry J, Jeremic B. Effects of prolongation of overall treatment time due to unplanned interruptions during radiotherapy of different tumor sites and practical methods for compensation. Int J Radiat Oncol Biol Phys. 2007; 68:654-661. [PubMed: 17467926]

23. Fromme EK, Kenworthy-Heinige T, Hribar M. Developing an easy-to-use tablet computer application for assessing patient-reported outcomes in patients with cancer. Support Care Cancer Off J Multinatl Assoc Support Care Cancer. 2011; 19:815-822.

24. Schag CC, Heinrich RL, Ganz PA. Karnofsky performance status revisited: reliability, validity, and guidelines. J Clin Oncol Off J Am Soc Clin Oncol. 1984; 2:187-193.

25. Hickman SE, Tilden VP, Tolle SW. Family reports of dying patients' distress: the adaptation of a research tool to assess global symptom distress in the last week of life. J Pain Symptom Manage. 2001; 22:565-574. [PubMed: 11516598]

26. Portenoy RK, Thaler HT, Kornblith AB, et al. The Memorial Symptom Assessment Scale: an instrument for the evaluation of symptom prevalence, characteristics and distress. Eur J Cancer Oxf Engl 1990. 1994; 30A:1326-1336.

27. Krebs EE, Carey TS, Weinberger M. Accuracy of the pain numeric rating scale as a screening test in primary care. J Gen Intern Med. 2007; 22:1453-1458. [PubMed: 17668269]

28. Fairclough DL, Cella DF. Functional Assessment of Cancer Therapy (FACT-G): non-response to individual questions. Qual Life Res Int J Qual Life Asp Treat Care Rehabil. 1996; 5:321-329.

29. Davis FD. Perceived usefulness, perceived ease of use, and user acceptance of information technology. MIS Q. 1989; 13:319-340.

30. Scoggins JF, Patrick DL. The use of patient-reported outcomes instruments in registered clinical trials: evidence from ClinicalTrials.gov. Contemp Clin Trials. 2009; 30:289-292. [PubMed: 19275948]

31. Blum D, Raj SX, Oberholzer R, et al. Computer-Based Clinical Decision Support Systems and Patient-Reported Outcomes: A Systematic Review. The Patient. 2014

32. Weiner M, Callahan CM, Tierney WM, et al. Using information technology to improve the health care of older adults. Ann Intern Med. 2003; 139:430-436. [PubMed: 12965971]

33. O'Connor AM, Rostom A, Fiset V, et al. Decision aids for patients facing health treatment or screening decisions: systematic review. BMJ. 1999; 319:731-734. [PubMed: 10487995] 


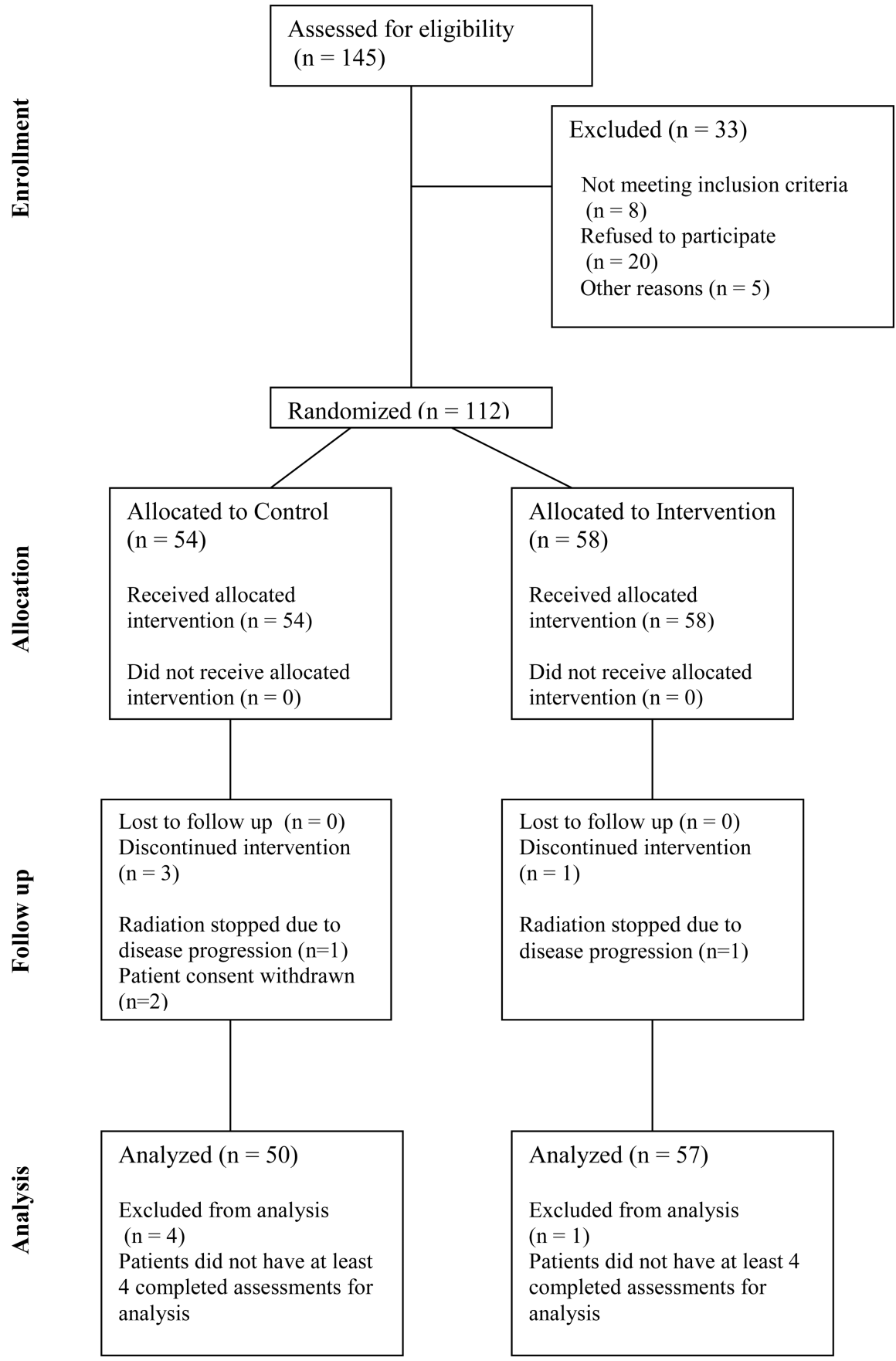

Figure 1.

CONSORT diagram for patients participating in a randomized, controlled pilot trial evaluating a computerized patient reported symptom assessment in radiotherapy. 

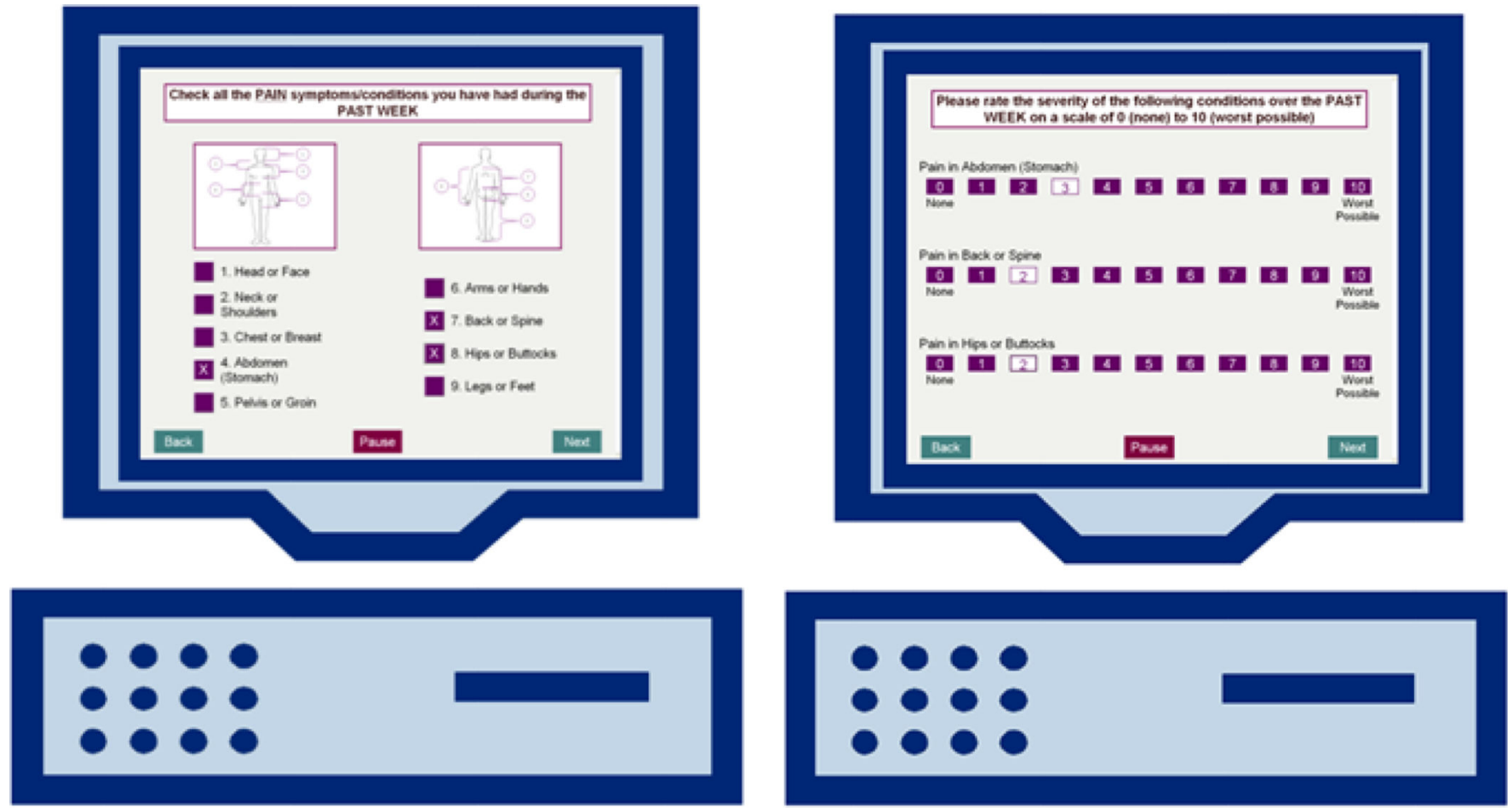

Figure 2.

Sample screen-shots showing the graphic user interface used by patients in both control and intervention arms to report symptoms prior to weekly on-treatment visits with their treating radiation oncologist. 


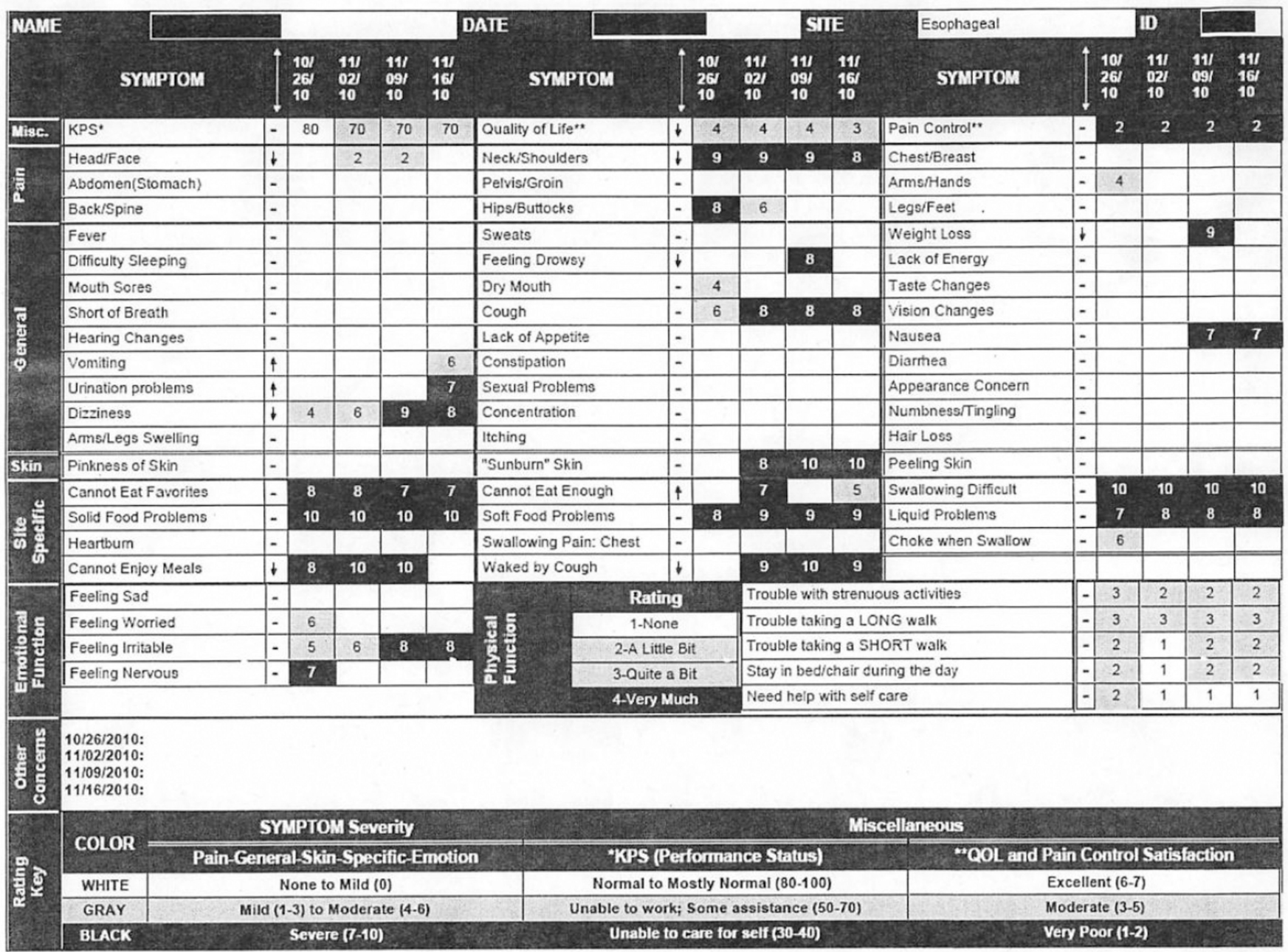

Figure 3.

Sample report showing what was printed and given to clinicians in the intervention groups. Patients received a version with larger font occupying two pages front and back. The top shows the survey dates and columns from left to right show how scores change over time. The first three rows show scores for Karnofsky Performance Status (KPS), Quality of Life (QOL) and satisfaction with pain control. The next three rows show scores for pain in different anatomic locations. The next nine rows show Memorial Symptom Assessment Scale (MSAS) scores. The following ten rows show skin toxicity symptoms, other sitespecific physical symptoms, and emotional and function scales. The bottom rows give a key to interpret the patient-reported outcomes (PROs) for symptoms, KPS, and QOL/Pain control satisfaction. 


\section{Control Group:}

$\mathrm{N}=26$ patients who consented to ontreatment-visit recording.

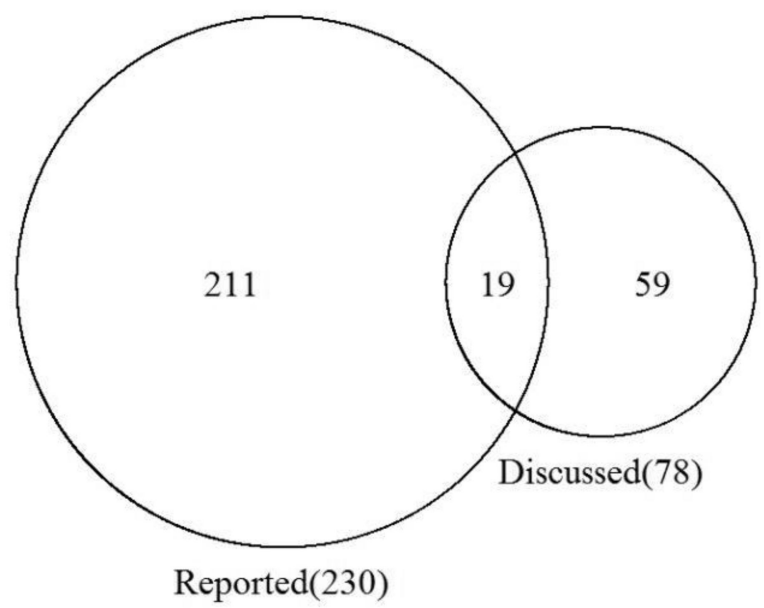

Intervention Group:

$\mathrm{N}=23$ patients who consented to ontreatment visit recording

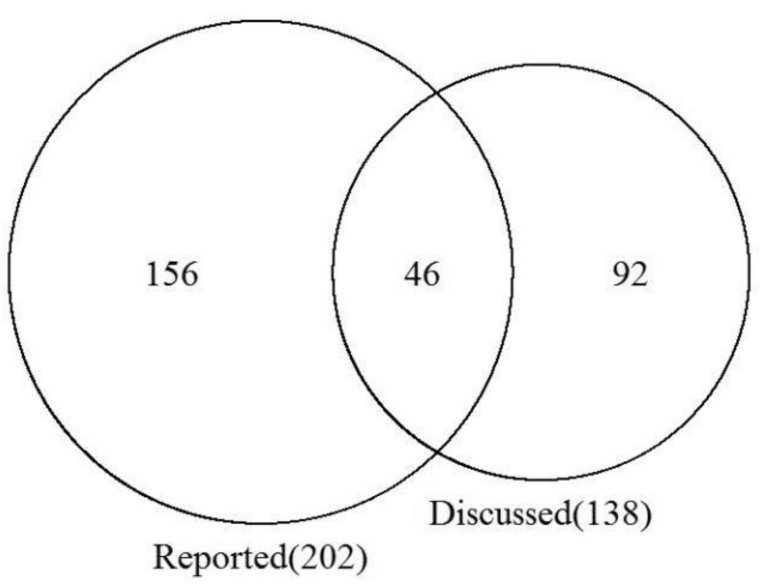

\section{All Symptoms}

\begin{tabular}{llll}
\hline & Intervention & Control & P-value \\
\hline Sensitivity accounting for within subject correlation & $23.17 \%$ & $10.28 \%$ & 0.03 \\
Sensitivity without accounting for within subject correlation & $22.70 \%$ & $8.30 \%$ & \\
\hline
\end{tabular}

Figure 4.

Venn Diagrams and sensitivity accounting to show concordance between patient-reported symptoms and symptoms discussed in weekly office visits for patients in the control and intervention groups. Because this analysis uses data from audiotaped patient-physician interactions, the samples size are control $n=26$ and intervention $n=23$. 
Table 1

Demographic information for patients the control and intervention arms

\begin{tabular}{|c|c|c|c|}
\hline $\mathrm{N}=112$ & $\begin{array}{c}\text { Control } \\
(\mathbf{n}=54) \\
\mathbf{n}(\%)\end{array}$ & $\begin{array}{c}\text { Intervention } \\
(\mathbf{n}=\mathbf{5 8}) \\
\mathbf{n}(\%)\end{array}$ & $P$ value ${ }^{*}$ \\
\hline Age (mean, SD) & $61.6(10.6)$ & $59.8(11.3)$ & 0.39 \\
\hline Female & $18(33.3 \%)$ & $14(24.1 \%)$ & 0.28 \\
\hline Non White/Caucasian & $5(9.3 \%)$ & $2(3.4 \%)$ & 0.32 \\
\hline \multicolumn{4}{|l|}{ Education } \\
\hline Bachelor Degree or higher & $22(46.8 \%)$ & $17(30.9 \%)$ & 0.43 \\
\hline \multicolumn{4}{|l|}{ Employment } \\
\hline Working Full or Part Time & $15(28.3 \%)$ & $20(34.5 \%)$ & 0.71 \\
\hline \multicolumn{4}{|l|}{ Tumor } \\
\hline Prostate & $23(42.6 \%)$ & $18(31.0 \%)$ & 0.07 \\
\hline Colorectal & $3(5.6 \%)$ & $10(17.2 \%)$ & \\
\hline Head \& Neck & $6(11.1 \%)$ & $14(24.1 \%)$ & \\
\hline Breast & $13(24.1 \%)$ & $7(12.1 \%)$ & \\
\hline Other & $9(16.7 \%)$ & $9(15.5 \%)$ & \\
\hline \multicolumn{4}{|l|}{ Tumor Stage } \\
\hline In situ & $3(5.6 \%)$ & $1(1.7 \%)$ & 0.49 \\
\hline I & $7(13.0 \%)$ & $13(22.4 \%)$ & \\
\hline II & $23(42.6 \%)$ & $19(32.8 \%)$ & \\
\hline III & $12(22.2 \%)$ & $14(24.1 \%)$ & \\
\hline IV & $9(16.7 \%)$ & $11(19.0 \%)$ & \\
\hline \multicolumn{4}{|l|}{ Oncology Treatment History } \\
\hline Concurrent Chemotherapy & $13(24.5 \%)$ & $25(43.1 \%)$ & 0.04 \\
\hline Previous Radiation & $6(11.3 \%)$ & $5(8.8 \%)$ & 0.66 \\
\hline \multicolumn{4}{|l|}{ Survey Session/Follow-up Exams } \\
\hline Completion of 4 survey sessions & $51(94.4 \%)$ & $57(100 \%)$ & 0.07 \\
\hline Continued surveys $\geq 5$ weeks & $38(70.4 \%)$ & $40(70.2 \%)$ & 0.95 \\
\hline \multicolumn{4}{|l|}{ Computer Use } \\
\hline Never used a touch screen computer & $12(22.2 \%)$ & $11(19.0 \%)$ & 0.36 \\
\hline \multicolumn{4}{|l|}{ Computer Experience } \\
\hline (Self-reported "Quite a Bit to Very Much") & $30(55.6 \%)$ & $31(53.4 \%)$ & 0.26 \\
\hline Willing to complete a Survey/Use a Summary as part of Usual Care & $41(80.4 \%)$ & $46(86.8 \%)$ & 0.92 \\
\hline
\end{tabular}

Support Care Cancer. Author manuscript; available in PMC 2017 April 01. 
* Pearson's Chi-Squared test was used for between-group comparisons of categorical variables, and Student's t-test was used for between-group comparisons of continuous variables.

SD-standard deviation. 
Table 2

Radiotherapy details for patients in the control and intervention arms

\begin{tabular}{|c|c|c|c|}
\hline $\mathrm{N}=112$ & $\begin{array}{l}\begin{array}{l}\text { Control } \\
(\mathbf{n}=54) \\
\mathbf{n}(\%)\end{array}\end{array}$ & $\begin{array}{l}\text { Intervention } \\
(\mathbf{n}=58) \\
\mathbf{n}(\%)\end{array}$ & $P$ value ${ }^{*}$ \\
\hline Radiotherapy Treatment & & & 0.39 \\
\hline IMRT & $53(98.2 \%)$ & $54(93.1 \%)$ & \\
\hline 3D CRT & $1(1.9 \%)$ & $3(5.2 \%)$ & \\
\hline 2D CRT & $0(0 \%)$ & $1(1.7 \%)$ & \\
\hline Elapsed Days; mean, (SD) & $42.1(9.1 \%)$ & $41.8(8.0 \%)$ & 0.82 \\
\hline Total Dose (cGy); mean, (SD) & $6290.3(918.8)$ & $6057.3(1272.5)$ & 0.27 \\
\hline Fraction Size (cGy); mean, (SD) & $212.3(30.6)$ & $205.3(27.3)$ & 0.20 \\
\hline Number of Fractions; mean (SD) & $29.4(5.6)$ & $29.9(5.7)$ & 0.67 \\
\hline Radiation Treatment Site & & & 0.47 \\
\hline Head/Neck & $10(18.5 \%)$ & $13(22.4 \%)$ & \\
\hline Pelvis & $27(50.0 \%)$ & $31(53.5 \%)$ & \\
\hline Abdomen & $3(5.7 \%)$ & $1(1.7 \%)$ & \\
\hline Breast & $11(20.4 \%)$ & $7(12.1 \%)$ & \\
\hline Chest & $3(5.6 \%)$ & $4(6.9 \%)$ & \\
\hline Other & $0(0.0 \%)$ & $2(3.5 \%)$ & \\
\hline Treatment Intent (Curative) & $51(94.4 \%)$ & $58(100 \%)$ & $\mathrm{p}=.98$ \\
\hline
\end{tabular}

* Pearson's Chi-Squared test was used for between-group comparisons of categorical variables.

IMRT-intensity-modulated radiation therapy, 3D CRT-3 dimensional conformal radiation therapy, 2D CRT-2 dimensional conformal radiation therapy, cGy-centigrey, SD-standard deviation. 


\section{Table 3}

Average Memorial Symptom Assessment Scale-Global Distress Index scores for patients in the control and intervention arms during weeks 1, 2, 3 and 4 of radiotherapy.

\begin{tabular}{|l|l|l|}
\hline $\mathbf{N}=112$ & $\begin{array}{l}\text { Control }(\mathbf{n}=54) \\
\text { Mean }(\mathbf{S D})\end{array}$ & $\begin{array}{l}\text { Intervention }(\mathbf{n}=58) \\
\text { Mean }(\mathbf{S D})\end{array}$ \\
\hline Week 1 & $072(0.64)$ & $0.86(0.68)$ \\
\hline Week 2 & $0.80(0.66)$ & $0.79(0.69)$ \\
\hline Week 3 & $0.83(0.73)$ & $0.83(0.66)$ \\
\hline Week 4 & $0.87(0.79)$ & $0.92(0.65)$ \\
\hline
\end{tabular}

SD-Standard Deviation 


\section{Table 4}

Multilevel model fixed effect results predicting the change in Memorial Symptom Assessment Scale Symptom Burden Global Distress Index over successive radiotherapy treatment weeks

\begin{tabular}{|c|c|c|}
\hline \multicolumn{3}{|l|}{ Global Distress Index $\S$} \\
\hline & B & SE \\
\hline Intercept $^{a}$ & $0.612^{* * *}$ & 0.102 \\
\hline Intervention group & 0.038 & 0.118 \\
\hline Age & -0.009 & 0.006 \\
\hline Gender & 0.002 & 0.133 \\
\hline Chemotherapy status & $0.439^{* * *}$ & 0.125 \\
\hline Linear Slope & 0.102 & 0.221 \\
\hline Intervention group & -0.055 & 0.256 \\
\hline Age & $0.026^{*}$ & 0.012 \\
\hline Gender & 0.220 & 0.289 \\
\hline Chemotherapy status & -0.304 & 0.272 \\
\hline Quadratic Slope & 0.005 & 0.075 \\
\hline Intervention group & 0.017 & 0.087 \\
\hline Age & $-0.008^{*}$ & 0.004 \\
\hline Gender & -0.105 & 0.097 \\
\hline Chemotherapy status & 0.047 & 0.092 \\
\hline
\end{tabular}

Note. Unstandardized coefficients are shown.

${ }^{a}$ The intercept has been coded to represent baseline scores.

$*$

$\mathrm{p}<.05$.

**

$\mathrm{p}<.01$.

*** $\mathrm{p}<.001$.


levels: within individuals (level one) and between individuals (level two). At level one, each patient's change in distress is represented by a trajectory captured by a set of unique parameters: intercept and slope. At level two, these unique parameters become outcome variables to be explained by a set of predictors. 